\title{
Oscillations and Velocity Structure of a Long-Lived Cyclonic Spot
}

\author{
artie Hatzes, Daniel D. Wenkert, ANDrew P. Ingersoll, G. EdWard Danielson
}

California Institute of Technology, Division of Geological and Planetary Sciences, Pasadena, California 91125

\begin{abstract}
Dark brown cyclonic spots ('barges') at $14^{\circ} \mathrm{N}$ were studied by using Voyager 1 and 2 images of Jupiter. Movie sequences were made to study the spots' behavior over intervals of 50 days and longer. These movies revealed that the length and width vary by $\pm 9 \%$ with a period of about 15 days, while the area remains approximately constant. The horizontal velocity field was investigated for an interval of about 1 day. Flow around the largest barge (feature 6) occurs as a ring current. The vorticity inferred is about $2 \frac{1}{2}$ times that of the ambient cyclonic zonal circulation, and about one-half the value of the local planetary vorticity. Length and width variations appear to be associated with a nonzero horizontal divergence field. If the oscillations are a natural mode of the system, the 15-day period will provide an important datum for testing models of stable closed vortices.
\end{abstract}

\section{INTRODUCTION}

Long-lived spots are common in Jupiter's atmosphere. Outside the equatorial zone they occur as regions of concentrated vorticity imbedded between counter-flowing east-west jets, which are also extremely long lived by terrestrial standards. Attempts to model this behavior are hampered by lack of data on velocity and temperature as functions of horizontal position, altitude, and time. In this paper, Voyager imaging data are used to obtain horizontal velocities as functions of horizontal position and time within and around a long-lived northern hemisphere spot. Although vertical structure and vertical velocity are largely unknown, the data reported here provide useful constraints on theoretical models.

The spots described here are an exception to the rule that cyclonic (low pressure) regions on Jupiter are disorganized and turbulent, whereas anticyclonic (high pressure) regions are compact, long lived, and stable [Ingersoll et al., 1979; Mitchell et al., 1979]. These features are brown oval spots (called 'barges') whose internal flow is cyclonic (counterclockwise in the northern hemisphere). They exist in a latitude band near $14^{\circ} \mathrm{N}$ where the ambient vorticity associated with the east-west flow is also cyclonic [see Figure 3 of Ingersoll et al., this issue]. The barges follow the general rule for Jupiter that cyclonic vorticity is associated with dark brown or blue color and high infrared radiances [Westphal et al., 1974; Terrile and Beebe, 1979; Hanel et al., 1979]. A plausible interpretation is that these are regions of depressed clouds, sinking motion, and low physical temperatures (i.e., lower temperatures than anticyclonic regions on constant pressure surfaces [Hess and Panofsky, 1951; Ingersoll and Cuzzi, 1969]). Color images of these barges have been published as Figure 5 of Smith et al. [1979a] and Figure 1 (features 6, 7, and 8) and Figure 4 of Smith et al. [1979b].

A property that makes these brown spots particularly interesting, and is reported here for the first time, is their oscillatory behavior. The Great Red Spot, an anticyclone in the southern hemisphere, changes its mean longitudinal position by $\pm 0.5^{\circ}$ with a 90 -day period [Solberg, 1969]. The Great Red Spot's oscillation is defined with respect to its average westward-drifting position, and amounts to a $\pm 2 \%$ movement compared to its major axis. In contrast, the largest of the barges (feature 6 in Figure 1 of Smith et al. [1979b]) changes its length and width with a 15-day period. The length shrinks as the width grows, and both vary by about $\pm 9 \%$, so that area is

Copyright (C) 1981 by the American Geophysical Union.

Paper number $80 \mathrm{C} 1753$

0148-0227/81/080C-1753\$01.00 approximately constant. Because the barge is smaller (major axis $\approx 7500 \mathrm{~km}$ ), a $\pm 2 \%$ oscillation in longitudinal position like the Great Red Spot's 90-day oscillation cannot be excluded at present.

\section{MeAsurements OF LeNGTH AND WIDTH}

The oscillation of feature 6 was discovered in time-lapse sequences of Voyager images. These sequences were put together from film products and were made into video movies covering over 25 days of observations. When possible, an image was selected from each Jovian rotation in which the feature was close to the central meridian of Jupiter's disk. Variations in longitude with respect to the central meridian were less than $10^{\circ}$ for this barge movie. Camera pointing uncertainties are removed by rerecording each film image onto video disk in sequence so that the feature of interest remains steady in the center of the screen. Although the scale increases dramatically as the spacecraft approaches Jupiter, oscillations and other time-dependent behavior are clearly seen. Encouraged by the apparent oscillations of the barge in the movie, measurements of the length and width of the barge were made from digital maps for a period covering roughly 50 days prior to Voyager 2 encounter. Voyager 2 data were chosen because coverage of the largest barge (feature 6) was better than Voyager 1 , and this feature remained fairly close to the central meridian for all measurements. By using the digital data, the pixel values, pixel locations, and distance in pixels between points were obtained in each picture. The range between the spacecraft and Jupiter was used to convert to distance in kilometers. Since all parts of the feature were within $15^{\circ}$ of the central meridian, and since the latitude of the spacecraft with respect to Jupiter did not change appreciably, the analysis was performed assuming a plane surface at infinite distance from the camera. Electronic and optical distortion within the Voyager cameras were negligible for these observations.

The length of a barge was defined as the major axis measured at the point of greatest contrast variation. The width was similarly defined, only for the minor axis. Because there were nonuniformities in the edge of the barge, several measurements were made of the width at various points far from the ends, and the average was taken.

Figures 1 and 2 show the length and width of feature 6 as functions of time from Voyager 2 images. Time is measured in Jovian rotations, where encounter occured on rotation 133. Rotation $R=0$ corresponds to FDS 19007.19, Picno 1672 J2$56, E R T=136$ days $2045: 39 ; 1979$. The oscillation is clearly 


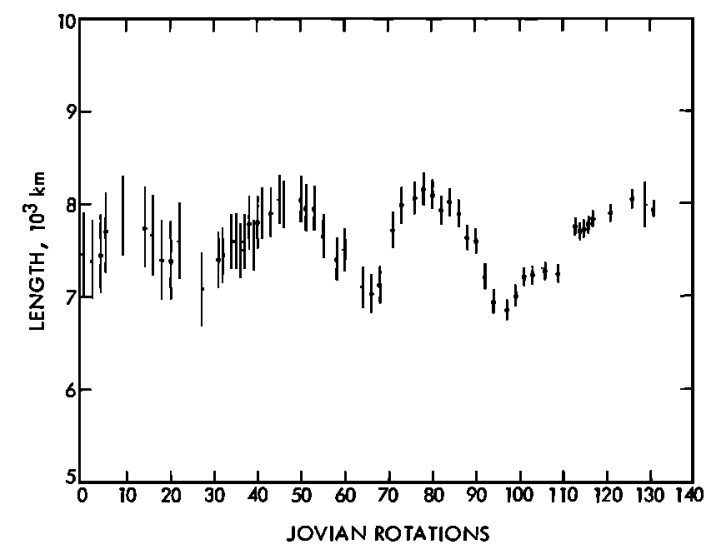

Fig. 1. The length of a brown oval (feature 6 of Smith et al. [1979b]) as a function of time. Rotation $=0$ has been arbitrarily chosen at FDS 19007.19. Encounter occurred during rotation 133.

evident. The maxima of Figure 1 are separated by intervals of $15,11.5$, and 20 days, respectively, indicating an irregular period. The duration of length minima seems to be less than that of length maxima. The maximum and minimum lengths are about 8200 and $6800 \mathrm{~km}$, respectively. The maximum and minimum widths are about 1600 and $1300 \mathrm{~km}$, respectively.

Comparison of Figures 1 and 2 reveals that the length and width oscillate approximately out of phase, suggesting that the area is constant. Direct measurements of the area were made for an interval covering 60 rotations (starting at $R=31$ ) by counting pixels in a computer printout of the digital image. Beyond this interval, starting at $R=90$, the area was determined by counting squares in a rectangular grid placed over a video image of the barge. In this way, measurements for the area were made almost up to encounter. Measurements prior to rotation 31 were not made because the images were small and the uncertainties large. The area of feature 6 is shown as a function of time in Figure 3. The area is approximately constant; any oscillations are less than $\pm 5 \%$ of the average area, which is about $9.9 \times 10^{6} \mathrm{~km}^{2}$. Thus, the fractional oscillations of area are less than half those of length or width. The area values also appear to decrease over the period of observation. This uniform decrease may be real or it may be a measurement effect due to the decreasing range and improved resolution in the pictures as the spacecraft approached Jupiter.

A total of two movies were made of this particular barge, one from Voyager 1 data and one from Voyager 2 data. Un-

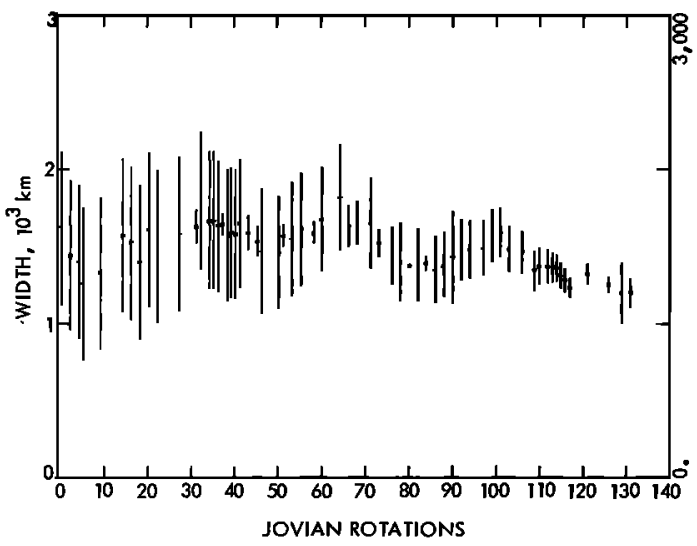

Fig. 2. Same as Figure 1 except that the width is plotted. Note that length and width oscillations are approximately out of phase.

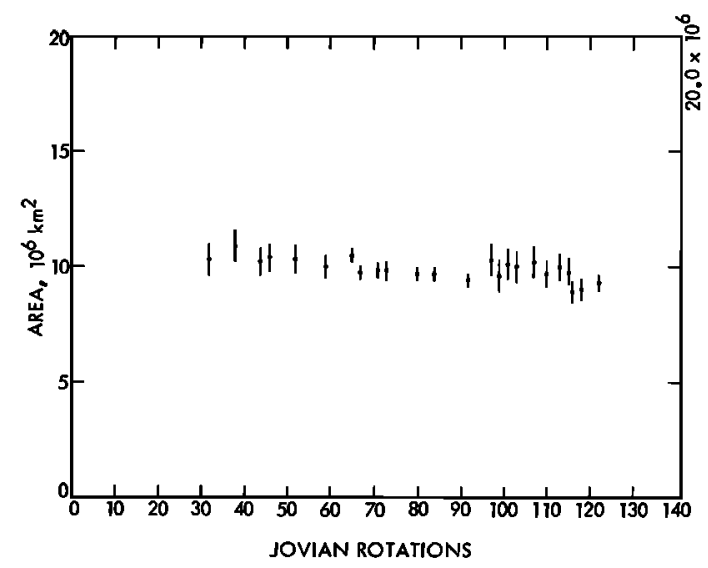

Fig. 3. Same as Figure 1 except that the area is plotted. Fractional oscillations in the area are less than one-half those of length or width.

like the Voyager 2 movie, the Voyager 1 movie did not readily show the 15-day oscillation, primarily because of inconsistent coverage and large variations in the position of the barge from the central meridian. The errors due to projection effects were so large that any oscillation was lost in the noise. However, when viewing the Voyager 1 movie one notices that the length and width change their size in relation to each other, but it is unclear whether this occurs in a periodic manner.

\section{MOTIONS ALONG BOUNDARY}

As is shown in Figure 3 of Beebe et al. [1980], the vorticity of the zonal currents at $13^{\circ}-15^{\circ} \mathrm{N}$ is about $1.2 \times 10^{-5} \mathrm{~s}^{-1}$. North of the barges the westward jet reaches a peak velocity of about 25-30 $\mathrm{m} \mathrm{s}^{-1}$. South of the barges the eastward jet reaches a peak velocity of about $100 \mathrm{~m} \mathrm{~s}^{-1}$. The barges themselves drift slowly eastward with the ambient flow at about 2.5 $\mathrm{m} \mathrm{s}^{-1}$ [Smith et al., 1979b].

Observations of spiral structures and chevron patterns around the barges and other rotating spots strongly suggest that they have more vorticity (rotate faster) than their surroundings [Ingersoll et al., 1979]. Linear features appear to be 'trailed behind' the core of any rapidly moving feature. Thus the direction of the spiral arms at the east and west ends of the barges [Figures 5 and 4 of Smith et al., 1979a, b, respectively] suggests that they have more cyclonic vorticity (counterclockwise rotation) than their surroundings. Direct observation of two small spots in the movie sequence yielded velocities of 20 $\pm 6 \mathrm{~m} \mathrm{~s}^{-1}$ for the counterclockwise flow around the periphery. Taking $18000 \mathrm{~km}$ for the circumference, $9.9 \times 10^{6} \mathrm{~km}^{2}$ for the area, and $20 \mathrm{~m} \mathrm{~s}^{-1}$ for the circumferential velocity, we obtain $3.6 \times 10^{-5} \mathrm{~s}^{-1}$ for the average vorticity, more than 3 times that of the ambient flow. The planetary vorticity $f=2 \Omega \sin \lambda$ at latitude $\lambda=14^{\circ}$ on Jupiter is $8.4 \times 10^{-5} \mathrm{~s}^{-1}$.

Another feature of the barges that became evident in the movie sequences is the presence of large-scale disturbances at the boundary. These appear as broad 'bumps' extending $1000 \mathrm{~km}$ along the boundary and $\sim 100 \mathrm{~km}$ out of the barge interior. They also move with the general counterclockwise flow around the periphery. Three of these disturbances were observed, and sequences of one of these disturbances is shown in Figure 4. Measured velocities are $15 \pm 10,24 \pm 8$, and $10 \pm$ $5 \mathrm{~m} \mathrm{~s}^{-1}$ for these disturbances, respectively. Within the uncertainties of observation, these velocities are not significantly different from that of the general flow defined by the smaller spots. Thus, it is not possible to say whether these distur- 


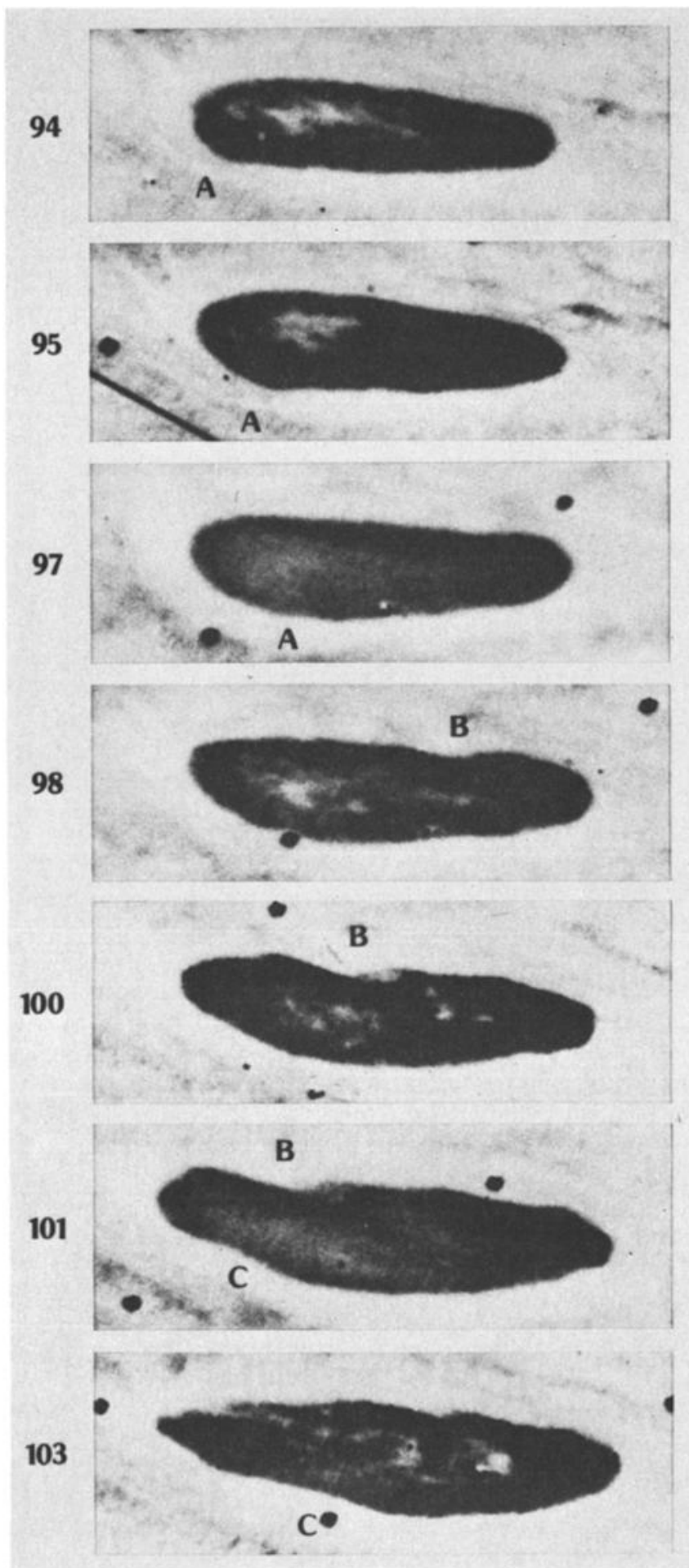

Fig. 4. Sequence showing propagation of disturbances (features A, B, C) during a minimum of the length oscillation cycle. Numbers are Jovian rotations, consistent with the other figures. Variability in cloud material in the oval's center can be seen in a time interval of 10 hours. Small dots are camera reseaus for measuring distortion.

bances are propagating waves or passive features that are carried by the general flow.

It is interesting that the time for a feature to circumnavigate the barge is $10-20$ days at the above-quoted velocities. This is essentially equal to the period of barge oscillations, as was discussed earlier. One possibility is that the oscillations are con- trolled by the vorticity distribution such that the natural frequency is equal to the average vorticity. Such a hypothesis could be used in testing a theoretical model of flow and stability of an elongated cyclone. Another possibility is that the barge oscillations are nothing more than the motion of an irregular boundary in a time-independent, regular, circumferential flow. According to the first hypothesis, the velocity field is varying with a 15 day period. According to the second hypothesis, only the boundary as defined by the cloud markings is varying. The velocity field itself may be steady.

From studying the movie sequences, we are inclined toward the first hypothesis. The barge oscillations look real. Moreover, the timing of interactions between disturbances and the barge oscillations is not consistent with the second hypothesis. If the apparent oscillations were merely due to motion of an irregular boundary, the length would be a maximum at times when outward bumps in the boundary reached the ends of the barge. A single bump would produce two maxima every time it circumnavigated the barge. Two bumps would produce four maxima, and so on. Thus the period of apparent oscillation would be less than 10 days, shorter than the observed period. Also, the movies contain examples of outward bumps starting at one end, when the barge length was a minimum and reaching the other end long before the length attains maximum. This tends to preclude the second hypothesis.

Rather, the oscillations appear to affect the formations and motion of the disturbances. These disturbances were formed at rotations 30,63 , and 94, respectively. Refering to Figure 1, one sees that these disturbances correspond very closely to the time when barge length was minimum. Also, the variations in the intervals between maxima in Figure $1(15,11.5$, and 20 days, respectively) are inversely related to the velocities (15, 24 , and $10 \mathrm{~m} \mathrm{~s}^{-1}$ ) inferred for the disturbances during these same periods.

\section{INSTANTANEOUS Velocity STRUCTURE}

Three Voyager images of the barge (from rotations 114, 115 , and 116) were analyzed as two matched pairs on the AMOS Interactive System [Yagi et al, 1978] at JPL to determine the horizontal velocities in and around the barge imaged in the movies. These images were selected because all three showed small wispy amounts of clouds within the barge, presumably at the same height in the atmosphere as those visible clouds around the barge. The horizontal velocities measured are presented as mapped vectors on the middle image (from rotation 115) in Figure 5. Careful analysis of this image shows (1) the flow around the barge is cyclonic, (2) the flow is concentrated as a ring current along the boundary of the barge, (3) meridonal velocities are significant only near the east and west ends of the barge, and (4) there is a strong north-south shear in zonal fiow throughout the barge.

In Figure 6, the zonal velocities of all measured points in a south to north slice through the barge (encompassing one-half of the barge's length) were plotted versus their latitudes. From this figure we estimate a zonal shear of $(\partial u / \partial y) \simeq-2.8( \pm 0.3)$ $\times 10^{-5} \mathrm{~s}^{-1}$. This is essentially the negative vertical component of vorticity, since in this region the meridional velocities are negligible. This vorticity is $\mathbf{2 . 3}$ times larger than the vorticity of the ambient cyclonic zonal circulation and is 3.1 times smaller than the local planetary vorticity.

Figure 7 presents the meridional velocities of all measured points in a west to east slice through the barge (encompassing 


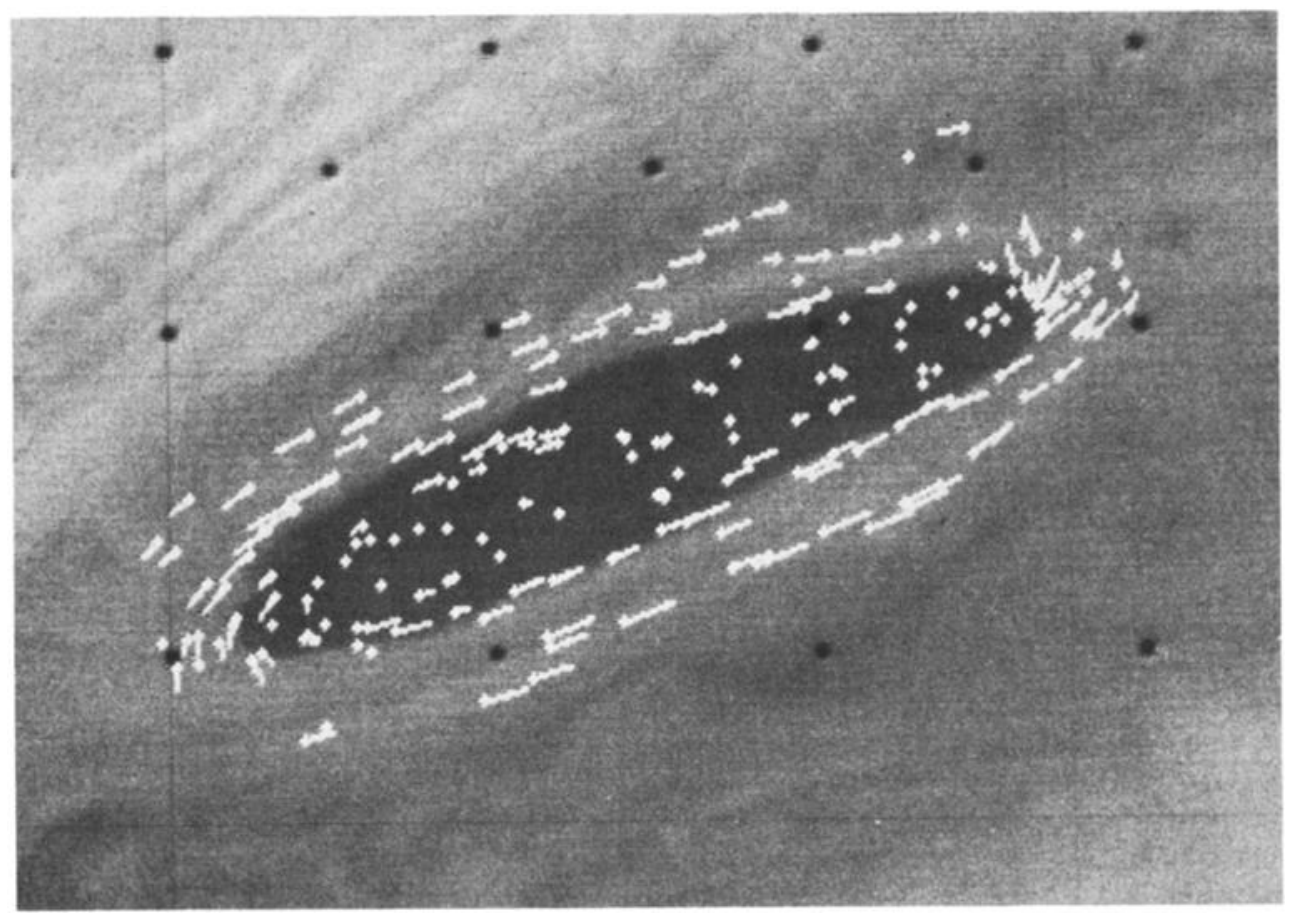

Fig. 5. Photo of barge (FDS 20433.15) with velocity vectors (as measured by using AMOS) plotted on it. Tails on vectors point in the direction of flow.

one-half of the barge's width) plotted versus their longitudes. Immediately obvious is the concentration of all meridional flow at the boundaries of the barge. The speed of the barge ring current at the east and west end of the barge seems to be about $25 \mathrm{~m} \mathrm{~s}^{-1}$. As seen in Figure 6, the zonal velocities at $12.5^{\circ} \mathrm{N}$ and $15^{\circ} \mathrm{N}$ are $53 \mathrm{~m} \mathrm{~s}^{-1}$ and $-41 \mathrm{~m} \mathrm{~s}^{-1}$, respectively. These are $21 \mathrm{~m} \mathrm{~s}^{-1}$ faster and slower, respectively, than the planetary average zonal velocities for these latitudes as given in Beebe et al. [1980]. Thus the boundary ring current of about

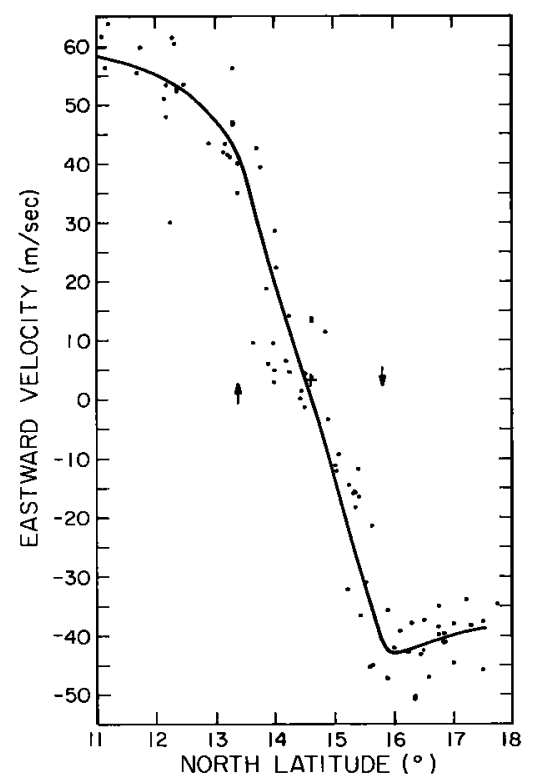

Fig. 6. Plot of all zonal velocities measured between longitudes $211.66^{\circ} \mathrm{W}$ and $218.02^{\circ} \mathrm{W}$ versus the latitudes at which the velocities were measured. The solid line is just an eyeball fit to the points, and the arrows show the northern and southern boundaries of the barge.
20-25 $\mathrm{m} \mathrm{s}^{-1}$ seems to be approximately constant around the perimenter of the barge.

The top half of Figure 8 shows a map of the vorticity field as determined from the AMOS data. Although the vorticity in the barge is roughly constant at about $2 \times 10^{-5} \mathrm{~s}^{-1}$, there are local maxima at the east and west ends of the barge. This is not surprising since the meridional flow seen near the ends adds its contribution to the vorticity due to the shear in zonal flow (which is seen throughout the barge). The divergence field as determined from the AMOS data is mapped in the bottom half of Figure 8 . Horizontal divergence in the barge is mostly quite small, although a pattern of alternating regions (going from west to east) of divergence and convergence can be discerned. Especially noticeable are the regions of positive

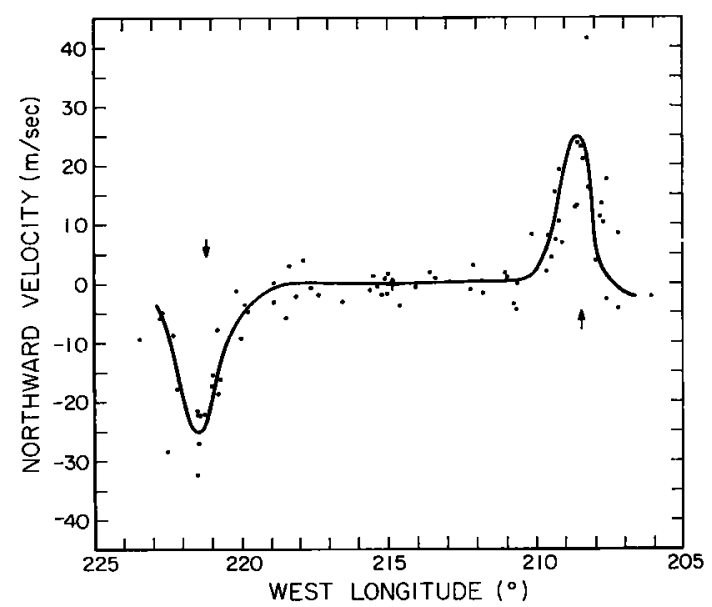

Fig. 7. Plot of all meridional velocities measured between latitudes $14.19^{\circ} \mathrm{N}$ and $15.00^{\circ} \mathrm{N}$ versus the longitudes at which the velocities were measured. The solid line is just an eyeball fit to the data, and the arrows show the eastern and western boundaries of the barge. 

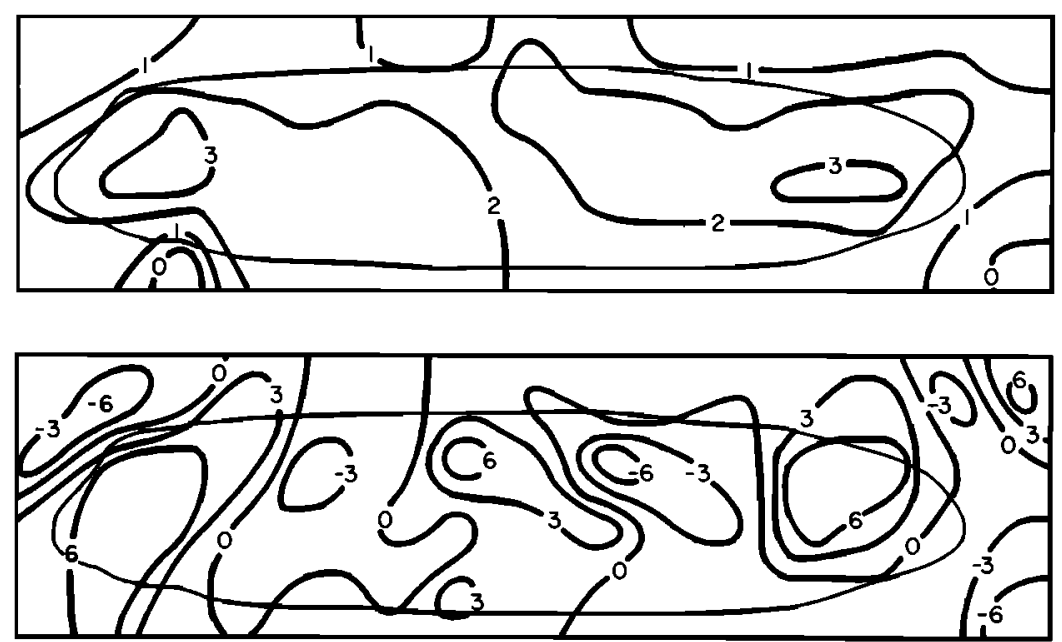

Fig. 8. (Top) A contour plot of the vertical component of vorticity computed from AMOS velocity data. The heavy lines are vorticity contours in $10^{-5} \mathrm{~s}^{-1}$, and the light line is an outline of the barge. (Bottom) A contour plot of the horizontal divergence field computed from AMOS velocity data. The heavy lines are divergence contours in $10^{-6} \mathrm{~s}^{-1}$, and the light line is an outline of the barge.

divergence (roughly $6 \times 10^{-6} \mathrm{~s}^{-1}$ ) at either end of the barge. Although the divergence is quite small compared to the vorticity, it is surprising that the regions of maximum positive divergence in the barge coincide with the regions of maximum cyclonic vorticity. It should be kept in mind, though, that these maps are from a time (as seen in Figure 1) in which the length is increasing rapidly. Apparently, there is some vertical flow associated with the oscillations.

In summary, the cyclonic brown spots at $14^{\circ} \mathrm{N}$ have values of vorticity about 2-3 times that of the surroundings. The length and width of the dark features oscillate with a 15-day period, the area remaining approximately constant. It is likely that this is a real dynamical oscillation (i.e., that the velocity field is also varying). If real, the 15-day period will be an important diagnostic for testing theoretical models.

Acknowledgment. Contribution number 3470 of the Division of Geological and Planetary Sciences, California Institute of Technology, Pasadena, California 91125.

\section{REFERENCES}

Beebe, R. F., A. P. Ingersoll, G. E. Hunt, J. L. Mitchel, and J.-P. Müller, Measurements of wind vectors, eddy momentum transports, and energy conversions in Jupiter's atmosphere from Voyager 1 images, Geophys. Res. Lett., 7, 1-4, 1980.

Hanel, R., B. Conrath, M. Flaser, L. Herath, V. Kunde, P. Lowman, W. Maguire, J. Pearl, J. Pirraglia, R. Samuelson, D. Gautier, P. Gierasch, L. Horn, S. Kumar, and C. Ponnamperuma, Infrared observations of the Jovian system from Voyager 2, Science, 206, 952956, 1979.

Hess, S. L., and H. A. Panofsky, The atmospheres of the other planets, in Compendium of Meteorology, pp. 391-400, American Meteorological Society, Boston, 1951.

Ingersoll, A. P., and J. V. Cuzzi, Dynamics of Jupiter's cloud bands, J. Atmos. Sci., 26, 981-985, 1969.
Ingersoll, A. P., R. F. Beebe, S. A. Collins, G. E. Hunt, J. L. Mitchell, P. Muller, B. A. Smith, and R. J. Terrile, Zonal velocity and texture in the Jovian atmosphere inferred from Voyager images, Nature, 280, 773-775, 1979.

Ingersoll, A. P., R. F. Beebe, J. L. Mitchell, G. W. Garneau, G. M. Yagi, and J.-P. Müller, Interaction of eddies and mean zonal flow on Jupiter as inferred from Voyager 1 and 2 images, $J$. Geophys. Res., this issue.

Mitchell, J. L., R. J. Terrile, B. A. Smith, J.-P Muller, A. P. Ingersoll, G. E. Hunt, S. A. Collins, and R. F. Beebe, Jovian cloud structure and velocity fields, Nature, 280, 776-778, 1979.

Smith, B. A., L. A. Soderblom, T. V. Johnson, A. P. Ingersoll, S. A. Collins, E. M. Shoemaker, G. E. Hunt, H. Masursky, M. H. Carr, M. E. Davies, A. F. Cook II, J. Boyce, G. E. Danielson, T. Owen, C. Sagan, R. F. Beebe, J. Veverka, R. G. Strom, J. F. McCauley, D. Morrison, G. A. Briggs, and V. E. Suomi, The Jupiter system through the eyes of Voyager 1, Science, 204, 951, $1979 a$.

Smith, B. A., L. A. Soderblom, R. Beebe, J. Boyce, G. Briggs, M. Carr, S. A. Collins, A. F. Cook II, G. E. Danielson, M. E. Davies, G. E. Hunt, A. Ingersoll, T. V. Johnson, H. Masursky, J. McCauley, D. Morrison, T. Owen, C. Sagan, E. M. Shoemaker, R. Strom, V. E. Suomi, and J. Veverka, The Galilean satellites and Jupiter: Voyager 2 imaging science results, Science, 206, 927-950, $1979 b$.

Solberg, H. G., Jr., A 3-month oscillation in the longitude of Jupiter's Red Spot, Planet Space Sci, 17, 1573-1580, 1969.

Terrile, R. J., and R. F. Beebe, Summary of historical data: Interpretation of the Pioneer and Voyager cloud configurations in a time-dependent framework, Science, 204, 948-951, 1979.

Westphal, J. A., K. Matthews, and R. J. Terrile, Five-micron pictures of Jupiter, Astrophys. J. Lett., 188, L111-L112, 1974.

Yagi, G., J. Lorre, and P. Jepsen, Dynamic feature analysis for Voyager at the Image Processing Laboratory, in Proceedings of the Conference on Atmospheric Environment of Aerospace Systems and Applied Meterology, Nov. 15-17, 1978, American Meterological Society, Boston, Mass., 1978.

(Received June 18, 1980;

revised December 1, 1980;

accepted December 8, 1980.) 